\title{
$\infty$
}

\section{Analysis of Polarization Properties of Optical Isolator for Fiber Laser}

\author{
Tae-Gon Kim \\ Department of Electrical and Electronic Engineering, Dongshin University Graduate School, Naju 520-714, Korea \\ Min-Woo Cheon ${ }^{\dagger}$ and Yong-Pil Park \\ Department of Biomedical Engineering, Dongshin University, Naju 520-714, Korea
}

Kyung-Jae Cho and Sung-Hak Kang

Fiber \& Networking Co., LTD., Gwangju 500-706, Korea

Received August 23, 2011; Revised September 22, 2011; Accepted September 28, 2011

\begin{abstract}
An isolator transmits light in the forward direction and blocks light from passing in the reverse direction. It is regarded an essential optical component in medical, industrial, and research lasers for blocking reflection beams that cause optical damage and noise. It is also used as a communicative light intensifier to expand the lifespan of devices and enhance transmission quality. This study analyzed the characteristics of the core components in the construction of a polarization-independent isolator, namely, the walk-off polarizer and the Faraday rotator. Measurement of the extinction ratio of the resultant walk-off polarizer revealed that the ratio between the vertical and horizontal rays was $1,050: 1$ with a laser output of $0.032 \mathrm{~W}$ and 1,010:1 with a laser output of $2.68 \mathrm{~W}$, thus presenting ratios similar to 1,000:1. In addition, the walk-off polarizer and Faraday rotator constructed in this study were used to compare output changes according to changes in power of input light and to check the penetration ratio. Results from the study presented variations in output value according to changes in power of input light. However, the average penetration ratio remained relatively consistent $(\sim 81.4 \%)$.
\end{abstract}

Keywords: Fiber laser, Isolator, Polarizer, Polarization beam splitter, Extinction ratio

\section{INTRODUCTION}

The structure of the laser produced using the optical fiber can be classified into the module and delivery components. The optical isolator is attached at the end of the delivery component of the laser.

The optical isolator permits only forward transmission of light, and prevents transmission in the backward direction. It is hence regarded an essential optical component in medical, industrial, and research lasers for blocking reflection beams that cause optical damage and noise. It is also used as a communica-

${ }^{\dagger}$ Author to whom all correspondence should be addressed:

E-mail: mwcheon@dsu.ac.kr

Copyright $@ 2011$ KIEEME. All rights reserved.

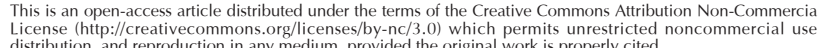
dstribution, and reprod tion in any medium, provided the original work is properly cited. tive light intensifier to expand the lifespan of devices and enhance transmission quality [1-3]. Optical isolators can be classified into two types: polarization-dependent and polarizationindependent [4]. The former type functions as an isolator only when the transmitted light presents a consistent polarization direction, while the latter can function as an isolator regardless of the direction of the transmitted light. The polarizationdependent type is commonly used in a laser diode whereas the polarization-independent type is used in an optical fiber intensifier.

This study used the walk-off phenomenon $[5,6]$ of extraordinary rays in birefractive materials to validate the characteristics of the Faraday rotator with the help of a walk-off polarizer and a magneto-optical (MO) glass to produce a polarization-independent isolator. 


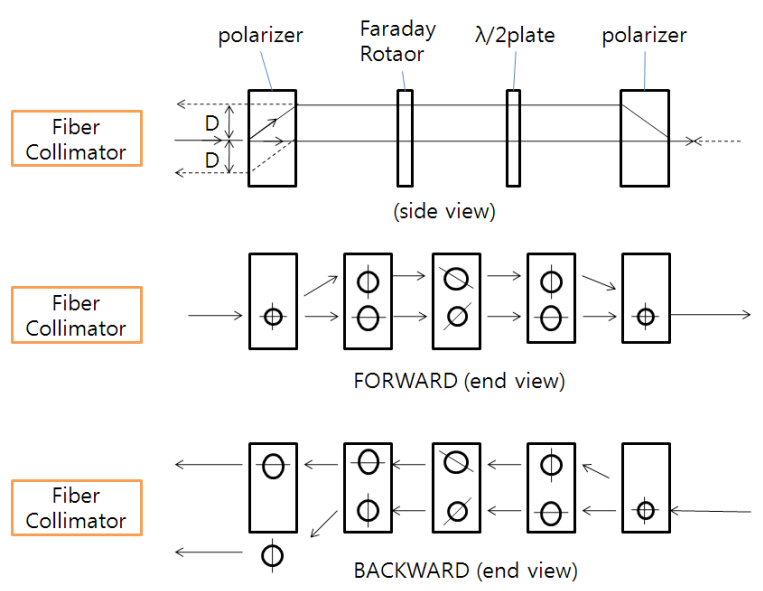

Fig. 1. Walk-off polarization principle.

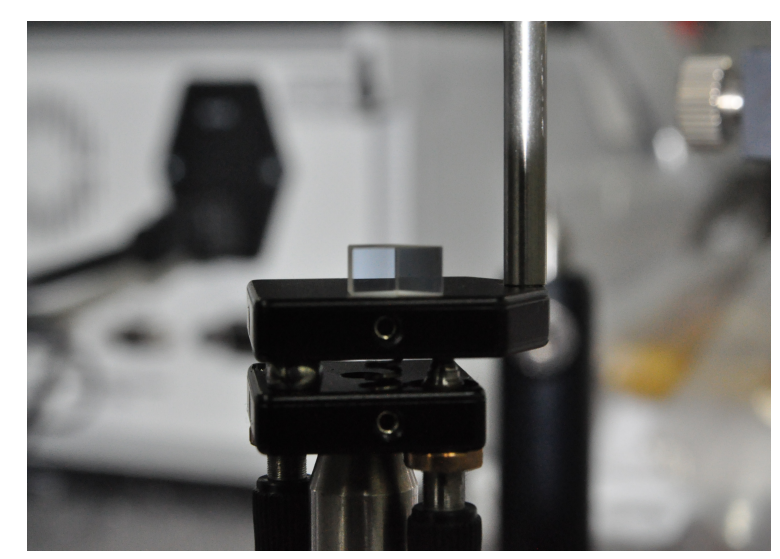

Fig. 2. Walk-off polarizer.

\section{EXPERIMENTS}

\subsection{Walk-off polarizer}

The polarizer plays an important role in determining the performance of the optical isolator as it permits transmission of substances parallel to the reflective side, whereas it reflects substances that are not parallel to the reflective side during transmission of the incident laser. On one hand, laser transmitted through the optical isolator is divided into two paths after passing through the first polarizer, and once the direction of the polarized light is rotated $90^{\circ}$, directly moving light passes by the Faraday rotator to be focused on the analyzer, that is, the second polarizer, for output. On the other hand, light moving in the reverse direction first passes the analyzer to be divided into an ordinary and an extraordinary ray. It does not affect the optical fiber that uses the light source. This is because a phase difference of $90^{\circ}$ is formed with the direction of the polarized light during forward propagation of light as $45^{\circ}$ rotations are made when moving by the Faraday rotator and MO material. The walk-off polarization principle of the optical isolator is shown in Fig. 1.

The MO glass used in the Faraday rotator in this study selected a walk-off distance of $4 \mathrm{~mm}$ in consideration of the 10 - $\mathrm{mm}$ diameter and beam size of the fiber laser. The produced walk-off polarizer is presented in Fig. 2.

The produced walk-off polarizer was designed with a 1,000:1 extinction ratio to penetrate vertical polarization materials and reflect horizontal polarization materials. In addition, a polarization beam splitter (PBS) that can withstand a large output of up

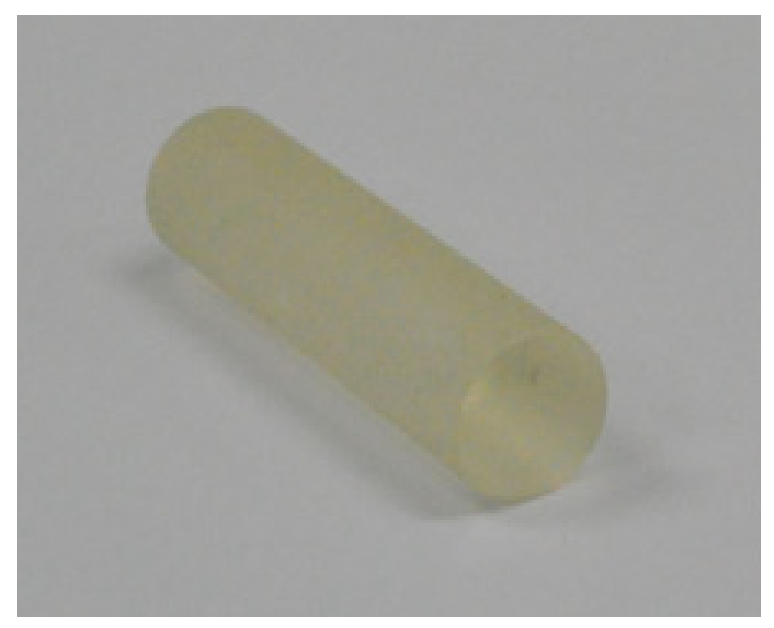

Fig. 3. Magneto-optical glass.

to $10 \mathrm{~W}$ was used to maintain the 4 -mm beam walk-off distance. The polarizer was designed and produced in sizes of $8 \times 4 \times 4$ $\mathrm{mm}$.

\subsection{Faraday rotator}

To rotate the polarized light by $45^{\circ}$ using the nonreciprocal method, the Faraday rotator that is used to generate the phase difference in the light incident on the isolator needs an MO glass with a large proportional factor value and a permanent magnet for generating an appropriate magnetic field [7-10]. Various MO materials have been developed for the Faraday rotator including optical crystals, such as terbium gallium garnet (TGG) and yttrium iron garnet (YIG), rare earth-additive silica-optical glass, such as FR5 and MOG10, glycogen-optical glass, such as As-Se [11], and films such as (TbBi)3(FeAl)5O12. However, TGG and $\mathrm{Tb}$-doped silicate glass are being used for large outputs of more than $10 \mathrm{~W}$ level. For the MO glass, this study used MR3-2, a lowcost terbium-doped borosilicate glass that has a high optical constant, low absorption coefficient, low nonlinear refractive index, and high damage threshold for laser energy. The MO glass used in this study is shown in Fig. 3.

\section{RESULTS AND DISCUSSION}

\subsection{Walk-off polarizer}

Magnetic substances used in the generation of the magnetic field include the alnico magnet, ferrite magnet, samarium-cobalt magnet, and neodymium magnet. This study used the neodymium magnet, which is mainly composed of rare-earth elements, pure iron, and boron. This magnet has relatively stronger magnetism and can be processed more easily when compared with the samarium-cobalt magnet.

The walk-off polarizer was produced using PBS to penetrate the vertical polarization substances and reflect the horizontal polarization substances. The performance of the walk-off polarizer was evaluated by conducting an extinction ratio test on the polarizer using an Nd:YAG laser of 1,064 nm wavelength . A simple schematic diagram representing the extinction ratio test of the polarizer is shown in Fig. 4.

The extinction ratio test is an experiment for measuring the performance of the polarizer in distributing the vertical and horizontal substances of the laser. To accurately determine the laser polarization status, the experiment was designed such that a fil- 


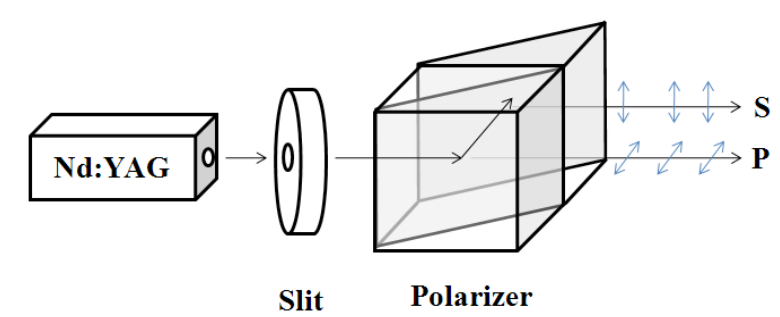

Fig. 4. Extinction ratio test of the polarizer.

Table 1. Extinction ratios test result.

\begin{tabular}{cccc}
\hline Laser power & Ordinary rays & $\begin{array}{c}\text { Extraordinary } \\
\text { rays }\end{array}$ & Extinction ratio \\
\hline \hline $0.03225 \mathrm{~W}$ & $0.032 \mathrm{~W}$ & $0.00025 \mathrm{~W}$ & $1,050: 1$ \\
$2.682 \mathrm{~W}$ & $2.68 \mathrm{~W}$ & $0.002 \mathrm{~W}$ & $1,010: 1$ \\
\hline
\end{tabular}

Table 2. Degree of polarization versus wavelength.

\begin{tabular}{cccc}
\hline Magnet & \multirow{2}{*}{ Structure } & \multicolumn{2}{c}{ Degree of polarization } \\
\cline { 3 - 4 }$($ diameter $\times$ bore $\times$ length) & & $632.8 \mathrm{~nm}$ & $1,064 \mathrm{~nm}$ \\
\hline $60 \varphi \times 11 \varphi \times 40 \mathrm{~mm}(1 \mathrm{EA})$ & Mixed block & $225^{\circ}$ & $75^{\circ}$ \\
$60 \varphi \times 11 \varphi \times 20 \mathrm{~mm}(2 \mathrm{~A})$ & & & \\
$60 \varphi \times 11 \varphi \times 40 \mathrm{~mm}(1 \mathrm{EA})$ & Single block & $135^{\circ}$ & $45^{\circ}$ \\
$60 \varphi \times 11 \varphi \times 20 \mathrm{~mm}(1 \mathrm{EA})$ & Single block & $90^{\circ}$ & $30^{\circ}$ \\
$60 \varphi \times 15 \varphi \times 30 \mathrm{~mm}(1 \mathrm{EA})$ & Single block & $113^{\circ}$ & $37.67^{\circ}$ \\
$60 \varphi \times 15 \varphi \times 30 \mathrm{~mm}(2 \mathrm{EA})$ & Mixed block & $114^{\circ}$ & $38^{\circ}$ \\
$26 \varphi \times 16 \varphi \times 30 \mathrm{~mm}(1 \mathrm{EA})$ & Single block & $33^{\circ}$ & $11^{\circ}$ \\
\hline
\end{tabular}

ter was used to extract only the vertical polarization substances, and the polarization status was converted to measure the maximum point of reflected or penetrated light. A slit was used to transmit the measured light source into the laser power meter (LaserStar; Ophir Corporation, Littleton, CO, USA) to prevent reflected or penetrated light from affecting measurement during experiment. The output of the laser was measured between 0.03225 and $2.682 \mathrm{~W}$. The results of the extinction ratio test using the walk-off polarizer are presented in Table 1. The extinction ratios of the ordinary and extraordinary rays generated using the walk-off polarizer were verified to be approximately 1,000:1.

\subsection{Evaluation of the faraday rotator characteristics}

The permanent magnet is an important component of the Faraday rotator that affects its performance and determines the size of the optical isolator. The performance and size of the Faraday rotator are in turn largely dependent on the strength and characteristics of the permanent magnet. The permanent magnet used in the Faraday rotator is composed either of a single-block structure using one permanent magnet or of a complex-block structure produced by integrating various pieces of single blocks. As the complex-block structure can considerably increase the magnetic field strength, it can be used as a miniature optical device and is thus being frequently developed for this use.

This study used an MO glass of diameter $10 \varphi$ and length 37 $\mathrm{mm}$ as the MO material of the Faraday rotator. Permanent magnets of 4 standards such as the following: (1) $60 \varphi$ external diameter $\times 11 \varphi$ internal diameter $\times 40 \mathrm{~mm}$ length, (2) $60 \varphi$ external diameter $\times 11 \varphi$ internal diameter $\times 20 \mathrm{~mm}$ length, (3) $60 \varphi$ external diameter $\times 15 \varphi$ internal diameter $\times 30$-mm length (4) $26 \varphi$ external diameter $\times 16 \varphi$ internal diameter $\times 30 \mathrm{~mm}$ length were produced and a single or complex block was composed to install in the magnet holder. The degree of polarization was measured

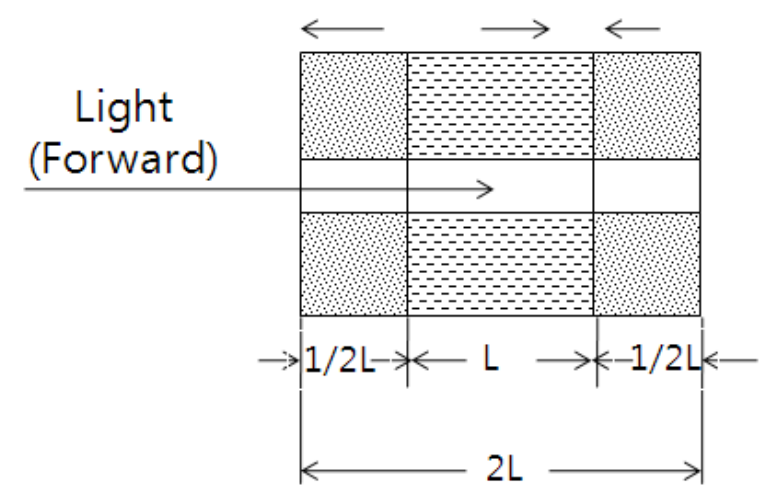

Fig. 5. The permanent magnet composition of the complex-block structure.

Table 3. Loss measurement results.

\begin{tabular}{ccc}
\hline Input power $(\mathrm{mW})$ & Output power $(\mathrm{mW})$ & Transmission $(\%)$ \\
\hline \hline 100 & 82.1 & 82.1 \\
200 & 162.4 & 81.2 \\
250 & 244.1 & 81.3 \\
300 & 324.5 & 81.1 \\
\hline
\end{tabular}

for two wavelengths: 632.8 and 1,064 nm.

In the initial condition without the Faraday rotator, the polarization status of the polarization analyzer (TXP 5004; Thorlabs Inc., Newton, NJ, USA) was set to $0^{\circ}$. The rotator was then fastened to the center of each magnetic substance and the degree of rotation was measured from $0^{\circ}$. As a result, changes in polarization angle could be verified according to the strength and wavelength of the magnetic field. In particular, a higher angle of polarization was observed in the complex-block magnetic structure. Thus, this study used a neodymium magnet of $60 \varphi$ external diameter, $11 \varphi$ internal diameter, and $40 \mathrm{~mm}$ length to make the Faraday rotator and applied this rotator to obtain a $45^{\circ}$ polarization in the 1,064 nm wavelength $\mathrm{Nd}$ :YAG laser.

\subsection{Measurement of penetration ratio}

The penetration ratio was measured using the produced polarizer and Faraday rotator. An Nd:YAG laser was used for the experiment, and the input power was increased from $100 \mathrm{~mW}$ to $400 \mathrm{~mW}$ in units of $100 \mathrm{~mW}$. The output was measured by passing the produced polarizer and Faraday rotor in the laser power meter. Results are presented in Table 3.

The power of the input laser beam was increased to increase the output obtained, and the results show that the average penetration rate was approximately $81.4 \%$.

\section{CONCLUSIONS}

The optical isolator is used to enhance the protection and safety of devices and finds applications in the optical fiber laser and optical communication system. This study analyzed the characteristics of the core components of the isolator, namely, the walk-off polarizer and the Faraday rotator, to produce a polarization-independent isolator.

The walk-off polarizer used PBS to endure a large output of up to $10 \mathrm{~W}$ and was designed and produced to present a spectrum of horizontal and vertical rays in 1,000:1 ratio. Based on the measurement of the extinction ratios of the produced walk-off polarizer, the ratio of the vertical and horizontal rays was measured to 
be 1,050:1 with $0.032 \mathrm{~W}$ laser output and 1,010:1 with $2.68 \mathrm{~W}$ laser output, thus presenting ratios similar to 1,000:1.

Terbium-doped borosilicate glass (MR3-2) used for the MO substance of the Faraday rotator and various forms of permanent magnets produced in neodymium were used to analyze the characteristics of the Faraday rotor. The experiment applied $632.8 \mathrm{~nm}$ and 1,064 nm light in various forms of single or complex-block magnetic fields to measure the degree of polarization versus the wavelength. Finally, the Faraday rotator composed using the single-block neodymium magnet of $60 \varphi$ external diameter, $11 \varphi$ internal diameter and $40 \mathrm{~mm}$ length was used to obtain $45^{\circ}$ polarization of the 1,064 $\mathrm{nm}$ wavelength light used in the isolator. The walk-off polarizer and Faraday rotator produced as a result of the study were used to compare the changes in output on the basis of changes in power of the input light and to check the penetration ratio. The experimental results showed variations in output value according to changes in power of input light. However, the average penetration ratio remained relatively consistent $(\sim 81.4 \%)$.

\section{ACKNOWLEDGMENTS}

This work was supported by the Ministry of Knowledge Economy.

\section{REFERENCES}

[1] J. W. Roh, J. S. Yang, W. Y. Lee, S. H. Ok, D. H. Woo, Y. T. Byun, T. M. Jhon, and S. Lee, J. Korean Inst. Mater. 43, 703 (2005).

[2] J. S. Tang, J. Korean Magn. Soc. 15, 148 (2005).

[3] R. M. Jopson, G. Eisenstein, H. E. Earl, and K. L. Hall, Electron. Lett. 21, 783 (1985) [http://dx.doi.org/10.1049/el:19850552].

[4] J. S. Jeon, J. Opt. Soc. (Korea) 8, 37 (1997).

[5] M. Shirasaki and K. Asama, Appl. Opt. 21, 4296 (1982) [http:// dx.doi.org/10.1364/AO.21.004296].

[6] K. W. Chang and W. V. Sorin, Opt. Lett. 15, 449 (1990) [http:// dx.doi.org/10.1364/OL.15.000449].

[7] A. Popkov, M. Fehndrich, M. Lohmeyer, and H. Dotsch, Appl. Phys. Lett. 72, 2508 (1998) [http://dx.doi.org/10.1063/ 1.121402].

[8] N. Bahlmann, V. Chandrasekhara, A. Erdmann, R. Gerhardt, P. Hertel, R. Lehmann, D. Salz, F. J. Schroteler, M. Wallenhorst, and H. Dotsch, J. Lightwave Technol. 16, 818 (1998) [http:// dx.doi.org/10.1109/50.669010].

[9] H. Yokoi and T. Mizumoto, Electron. Lett. 33, 1787 (1997) [http://dx.doi.org/10.1049/el:19971253].

[10] T. Mizumoto, H. Chihara, N. Tokui, and Y. Naito, Electron. Lett 26, 199 (1990) [http://dx.doi.org/10.1049/el:19900134].

[11] H. Sato, M. Kawase, and M. Saito, Appl. Opt. 24, 2300 (1985) [http://dx.doi.org/10.1364/AO.24.002300]. 